\title{
AN EPIGRAPHICAL CONVEX OPTIMIZATION APPROACH FOR MULTICOMPONENT IMAGE RESTORATION USING NON-LOCAL STRUCTURE TENSOR
}

\author{
Giovanni Chierchia ${ }^{1}$, Nelly Pustelnik ${ }^{2}$, Jean-Christophe Pesquet ${ }^{3}$, and Béatrice Pesquet-Popescu ${ }^{1}$ \\ ${ }^{1}$ Télécom ParisTech/Institut Télécom, LTCI, UMR CNRS 5141, 75014 Paris \\ ${ }^{2}$ Laboratoire de Physique - ENSL, UMR CNRS 5672, F-69007 Lyon, France \\ ${ }^{3}$ Université Paris-Est, LIGM, UMR CNRS 8049, 77454 Marne-la-Vallée, France
}

\begin{abstract}
TV-like constraints/regularizations are useful tools in variational methods for multicomponent image restoration. In this paper, we design more sophisticated non-local TV constraints which are derived from the structure tensor. The proposed approach allows us to measure the non-local variations, jointly for the different components, through various $\ell_{1, p}$ matrix norms with $p \geq 1$. The related convex constrained optimization problems are solved through a novel epigraphical projection method. This formulation can be efficiently implemented thanks to the flexibility offered by recent primal-dual proximal algorithms. Experiments carried out for color images demonstrate the interest of considering a Non-Local Structure Tensor TV and show that the proposed epigraphical projection method leads to significant improvements in terms of convergence speed over existing numerical solutions.
\end{abstract}

Index Terms - Convex optimization, color image restoration, non-local total variation, structure tensor, singular value decomposition.

\section{INTRODUCTION}

This work deals with the restoration of multicomponent images, such as color images, by adopting a constrained convex optimization approach. Such a formulation may be preferred to a regularized one since it has been recognized for a long time that incorporating constraints directly on the solution often facilitates the choice of the involved parameters $[1,2,3,4,5]$. Indeed, the constraint bounds are usually related to some physical properties of the target solution or to some knowledge of the degradation process, e.g. the noise statistical properties. One of the difficulties of constrained approaches is however that a closed form of the projection onto the considered constraint set is not always available. Closed forms are known for convex sets such as $\ell_{2}$-balls, hypercubes defining dynamics range constraints, or half-spaces. However, more sophisticated constraints are usually necessary in order to efficiently restore multicomponent images. Taking advantage of the flexibility offered by recent proximal algorithms, we propose an epigraphical method allowing us to address a wide class of convex constraints.

The quality of the results obtained through a variational approach strongly depends on the ability to model the regularity present in images. Since natural images are often piecewise smooth, popular regularization models tend to penalize the image gradient. In this context, Total Variation (TV) [6] has emerged as a simple, yet successful, convex optimization tool. However, TV fails to preserve textures, details and fine structures, because they are hardly distinguishable from noise. To improve this behaviour, the TV model has been extended by using a non-locality principle [7]. Another approach to overcome these limitations is to replace the gradient operator with a frame representation which yields a more suitable sparse representation of the image [8]. The connections between these two different approaches have been studied in [9]. It is still unclear which approach leads to the best results. However, there is some evidences that Non-Local (NL) TV may perform better in some image restoration tasks $[10,11]$. We thus focus our attention on NLTV-based constraints, although our proposed algorithm is quite general and it can also be adapted to frame-based approaches.

Related work and motivations The extension of TV-based models to multicomponent images is, in general, non trivial. A first approach consists of computing TV channel-by-channel and then summing up the resulting smoothness measures [12, $13,14,15,16]$. Since there is no coupling of the components, this approach may potentially lead to component smearing and loss of edges across components. An alternative way is to process the components jointly, so as to better reveal details and features that are not visible in each of the components considered separately. This approach naturally arises when the gradient of a multicomponent image is thought of as a structure tensor [17, 18, 19, 20, 21, 22]. A concise review of both frameworks can be found in [22].

The algorithmic solutions proposed in the aforementioned works are mostly based on PDEs [18, 19, 12, 20, 21], projected gradient methods $[14,15,16]$ or proximal algorithms such as FISTA or Chambolle-Pock primal-dual technique [22]. However, to the best of our knowledge, there does not exist an 
algorithm for dealing with structure tensor TV as a constraint. This is the main motivation of the present work.

Goal and contributions We propose a Structure Tensor (ST) NLTV in order to deal with multicomponent image recovery problems. This extends the ST-TV regularization proposed in the color restoration literature. Second, we provide an efficient solution based on proximal tools in order to solve convex problems involving matricial $\ell_{1, p}$-ball constraints. The proposed solution avoids the inner iterations that are implemented in the approaches in $[23,24]$ for solving regression problems. Outline The paper is organized as follows. Section 2 describes the degradation model and formulates the multicomponent constrained convex optimization problem based on a NL structure tensor. Section 3 explains how to minimize the corresponding objective function via proximal tools. Section 4 provides an experimental validation in the context of color image restoration. The conclusions are given in Section 5.

Notation $\Gamma_{0}\left(\mathbb{R}^{N}\right)$ denotes the set of proper, lower semicontinuous, convex functions from $\mathbb{R}^{N}$ to $\left.]-\infty,+\infty\right]$. The epigraph of $\varphi \in \Gamma_{0}\left(\mathbb{R}^{N}\right)$ is the nonempty closed convex subset of $\mathbb{R}^{N} \times \mathbb{R}$ defined as epi $\varphi=\left\{(y, \zeta) \in \mathbb{R}^{N} \times \mathbb{R} \mid \varphi(y) \leq \zeta\right\}$. The projection onto a nonempty closed convex subset $C \subset \mathbb{R}^{N}$ is, for every $y \in \mathbb{R}^{N}, P_{C}(y)=\operatorname{argmin}_{u \in C}\|u-y\|$.

\section{PROPOSED APPROACH}

Degradation model The $R$-component signal of interest is denoted by $\bar{x}=\left(\bar{x}_{1}, \ldots, \bar{x}_{R}\right) \in\left(\mathbb{R}^{N}\right)^{R}$. The degradation model is

$$
z=\mathcal{B}(A \bar{x})
$$

where $z=\left(z_{1}, \ldots, z_{S}\right) \in\left(\mathbb{R}^{K}\right)^{S}$ denotes the degraded observations, $A=\left(A_{j, i}\right)_{1 \leq j \leq S, 1 \leq i \leq R}$ is the degradation linear operator with $A_{j, i} \in \mathbb{R}^{K \times N}$ and $\mathcal{B}$ models the effect of a (non-necessarily additive) noise.

Criterion In order to recover $\bar{x}$ from the observations $z$, we use a variational approach that aims at solving the convex problem

$$
\underset{x}{\operatorname{minimize}} f(A x, z) \quad \text { s.t. } \quad\left\{\begin{array}{l}
x \in C \\
g(x) \leq \eta
\end{array}\right.
$$

where $\eta>0$ and $C$ denotes a closed convex subset of $\left(\mathbb{R}^{N}\right)^{R}$. The cost function $f(\cdot, z) \in \Gamma_{0}\left(\left(\mathbb{R}^{K}\right)^{S}\right)$ is related to the noise characteristics. For instance, $f$ is a quadratic function for additive Gaussian noise, an $\boldsymbol{\ell}_{1}$-norm when a Laplacian noise is involved, or a Kullback-Leibler divergence when dealing with Poisson noise. Function $g \in \Gamma_{0}\left(\left(\mathbb{R}^{N}\right)^{R}\right)$ is used to incorporate prior information. Some possible choices for this function have been mentioned in the introduction. In what follows, we introduce the proposed NL structure tensor constrained optimization problem.

Non-Local Structure Tensor We extend the tensor regularization in [22] to a Structure Tensor Non-Local TV constrained optimization. To do so, for every $\ell \in\{1, \ldots, N\}$, let us define the matrix

$$
X^{(\ell)}=\left(\omega_{\ell, n}\left(x_{i}^{(\ell)}-x_{i}^{(n)}\right)\right)_{n \in \mathcal{N}_{\ell}, 1 \leq i \leq R} \in \mathbb{R}^{M_{\ell} \times R}
$$

where $\mathcal{N}_{\ell}$ is a possibly non-local neighborhood of $\ell$ and $M_{\ell}$ denotes its size. Methods for building such a neigborhood and setting the associated weights $\left.\left(\omega_{\ell, n}\right)_{n \in \mathcal{N}_{\ell}} \in\right] 0,+\infty\left[{ }^{M_{\ell}}\right.$ are described in $[25,26,27,28]$. The resulting ST-NLTV constraint is

$$
g(x)=\sum_{\ell=1}^{N}\left\|X^{(\ell)}\right\|_{p}
$$

where $\|\cdot\|_{p}$ denotes the Schatten $p$-norm, with $p \geq 1$. Denoting by $\sigma_{X^{(\ell)}}=\left(\sigma_{X^{(\ell)}}^{(m)}\right)_{1 \leq m \leq \min \left\{M_{\ell}, R\right\}}$ the singular values of $X^{(\ell)}$ ordered in decreasing order, the case $p \in[1,+\infty[$ yields

$$
g(x)=\sum_{\ell=1}^{N}\left(\sum_{m=1}^{\min \left\{M_{\ell}, R\right\}}\left(\sigma_{X^{(\ell)}}^{(m)}\right)^{p}\right)^{1 / p}
$$

and $p=+\infty$ leads to

$$
g(x)=\sum_{\ell=1}^{N} \sigma_{X^{(\ell)}}^{(1)}
$$

When $p=1$, the Schatten norm reduces to the nuclear norm.

Note that the Structure Tensor TV (ST-TV) proposed in [22] is a special case of (4) that arises when, for every $\ell \in$ $\{1, \ldots, N\}, M_{\ell}=2, \mathcal{N}_{\ell}$ includes horizontal/vertical nearest neighbors, and, for every $n \in \mathcal{N}_{\ell}, \omega_{\ell, n}=1$.

\section{ALGORITHMIC SOLUTION}

Within the proposed constrained optimization framework, Problem (2) can be reformulated as follows:

$$
\underset{x}{\operatorname{minimize}} f(A x, z) \text { s.t. }\left\{\begin{array}{l}
x \in C, \\
F x \in D
\end{array}\right.
$$

where the set $C \subset\left(\mathbb{R}^{N}\right)^{R}$ can be used for example to constrain the dynamics range of the signal to be recovered. $F$ is the linear operator defined as

$$
F: x \mapsto\left[F_{1} x \ldots F_{N} x\right]
$$

where, for every $\ell \in\{1, \ldots, N\}, F_{\ell}$ is the linear operator that maps $x \in\left(\mathbb{R}^{N}\right)^{R}$ to the matrix $X^{(\ell)} \in \mathbb{R}^{M_{\ell} \times R}$ defined in (3). Hereabove, $D$ is the closed convex set defined as

$D=\left\{X=\left[X^{(1)^{\top}} \ldots X^{(N)^{\top}}\right]^{\top} \in \mathbb{R}^{M \times R} \mid \sum_{\ell=1}^{N}\left\|X^{(\ell)}\right\|_{p} \leq \eta\right\}$,

with $M=M_{1}+\cdots+M_{N}$. 
In recent works, iterative procedures have been proposed to deal with an $\ell_{1,2}$-ball constraint [23] or an $\ell_{1, \infty}$-ball constraint [24]. Similar techniques can be used to compute the projection onto $D$, but a more efficient method consists of using the epigraphical splitting method proposed in [28]. More precisely, we introduce an auxiliary vector $\zeta=\left(\zeta^{(\ell)}\right)_{1 \leq \ell \leq N} \in \mathbb{R}^{N}$ in Problem (7) and decompose the constraint $D$ in two convex sets: a union of epigraphs

$$
\begin{aligned}
E= & \left\{(X, \zeta) \in \mathbb{R}^{M \times R} \times \mathbb{R}^{N} \mid\right. \\
& \left.(\forall \ell \in\{1, \ldots, N\}) \quad\left(X^{(\ell)}, \zeta^{(\ell)}\right) \in \mathrm{epi}\|\cdot\|_{p}\right\},
\end{aligned}
$$

and a closed half-space

$$
W=\left\{\zeta \in \mathbb{R}^{N} \mid 1_{N}^{\top} \zeta \leq \eta\right\},
$$

with $1_{N}=(1, \ldots, 1)^{\top} \in \mathbb{R}^{N}$. By using the proposed epigraphical splitting method, Problem (7) can be recast as

$$
\underset{(x, \zeta)}{\operatorname{minimize}} f(A x, z) \quad \text { s.t. }\left\{\begin{array}{l}
x \in C, \\
(F x, \zeta) \in E, \\
\zeta \in W .
\end{array}\right.
$$

The projection onto $W$ is trivial, while the projection onto $E$ is given by the following new result:

Proposition 3.1 Let $\ell \in\{1, \ldots, N\}$ and let $X^{(\ell)} \in \mathbb{R}^{M_{\ell} \times R}$. Let $U^{(\ell)} S^{(\ell)}\left(V^{(\ell)}\right)^{\top}$ be the Singular Value Decomposition of $X^{(\ell)}$ where $\left(U^{(\ell)}\right)^{\top} U^{(\ell)}=\mathrm{I},\left(V^{(\ell)}\right)^{\top} V^{(\ell)}=\mathrm{I}$ and $S^{(\ell)}=$ $\operatorname{Diag}\left(\mathbf{s}^{(\ell)}\right)$ with $\mathbf{s}^{(\ell)}=\left(\sigma_{X^{(\ell)}}^{(m)}\right)_{1 \leq m \leq \min \left\{M_{\ell}, R\right\}}$. Then,

$$
\left(U^{(\ell)} T^{(\ell)}\left(V^{(\ell)}\right)^{\top}, \theta^{(\ell)}\right)=P_{\text {epi }\|\cdot\|_{p}}\left(X^{(\ell)}, \zeta^{(\ell)}\right),
$$

where $T^{(\ell)}=\operatorname{Diag}\left(\mathrm{t}^{(\ell)}\right)$ and

$$
\left(\mathrm{t}^{(\ell)}, \theta^{(\ell)}\right)=P_{\text {epi }\|\cdot\|_{p}}\left(\mathrm{~s}^{(\ell)}, \zeta^{(\ell)}\right) .
$$

The above result shows that the projection onto the epigraph of the $\ell_{1, p}$ matrix norm can be deduced from the projection onto the $\ell_{1, p}$ vector norm. It turns out that closed form expressions of the latter projection exist when $p \in\{1,2,+\infty\}$ [28]. For example, for every $\left(\mathbf{s}^{(\ell)}, \zeta^{(\ell)}\right) \in \mathbb{R}^{\min \left\{M_{\ell}, R\right\}} \times \mathbb{R}$,

$$
P_{\text {epi }\|\cdot\|_{2}}\left(\mathbf{s}^{(\ell)}, \zeta^{(\ell)}\right)= \begin{cases}(0,0), & \text { if }\left\|\mathbf{s}^{(\ell)}\right\|_{2}<-\zeta^{(\ell)} \\ \left(\mathbf{s}^{(\ell)}, \zeta^{(\ell)}\right), & \text { if }\left\|\mathbf{s}^{(\ell)}\right\|_{2}<\zeta^{(\ell)} \\ \beta^{(\ell)}\left(\mathbf{s}^{(\ell)},\left\|\mathbf{s}^{(\ell)}\right\|_{2}\right), & \text { otherwise, }\end{cases}
$$

where $\beta^{(\ell)}=\frac{1}{2}\left(1+\frac{\zeta^{(\ell)}}{\left\|\mathbf{s}^{(\ell)}\right\|_{2}}\right)$. Moreover,

$$
P_{\text {epi }\|\cdot\|_{\infty}}\left(\mathrm{s}^{(\ell)}, \zeta^{(\ell)}\right)=\left(\mathrm{t}^{(\ell)}, \theta^{(\ell)}\right),
$$

where, for every $\mathrm{t}^{(\ell)}=\left(\mathrm{t}^{(\ell, m)}\right)_{1 \leq m \leq \min \left\{M_{\ell}, R\right\}} \in \mathbb{R}^{\min \left\{M_{\ell}, R\right\}}$,

$$
\begin{aligned}
\mathrm{t}^{(\ell, m)} & =\min \left\{\sigma_{X^{(\ell)}}^{(m)}, \theta^{(\ell)}\right\}, \\
\theta^{(\ell)} & =\frac{\max \left(\zeta^{(\ell)}+\sum_{k=\bar{k}}^{\min \left\{M_{\ell}, R\right\}} \nu^{(\ell, k)}, 0\right)}{\min \left\{M_{\ell}, R\right\}-\bar{k}+2} .
\end{aligned}
$$

Hereabove, $\left(\nu^{(\ell, k)}\right)_{1 \leq k \leq \min \left\{M_{\ell}, R\right\}}$ is a sequence of reals obtained by sorting $\left(\sigma_{X^{(\ell)}}^{(m)}\right)_{1 \leq m \leq \min \left\{M_{\ell}, R\right\}}$ in ascending order (by setting $\nu^{(\ell, 0)}=-\infty$ and $\nu^{\left(\ell, \min \left\{M_{\ell}, R\right\}+1\right)}=+\infty$ ), and $\bar{k}$ is the unique integer in $\left\{1, \ldots, \min \left\{M_{\ell}, R\right\}+1\right\}$ such that

$$
\nu^{(\ell, \bar{k}-1)}<\frac{\zeta^{(\ell)}+\sum_{k=\bar{k}}^{\min \left\{M_{\ell}, R\right\}} \nu^{(\ell, k)}}{\min \left\{M_{\ell}, R\right\}-\bar{k}+2} \leq \nu^{(\ell, \bar{k})} .
$$

Note that the computation of the SVD can be avoided when $p=2$.

\section{NUMERICAL RESULTS}

In our experiments, the matrix $A$ is a decimated convolution which applies to each component an uniform blur followed by a random decimation. We have thus $R=S$. The operator $\mathcal{B}$ corresponds to an additive zero-mean white Gaussian noise with standard deviation $\alpha$. The fidelity term related to the noise neg-log-likelihood is $f=\|A \cdot-z\|_{2}^{2}$.

The experiments are focused on color imaging, i.e. the case $R=3$. While it is common for color imaging to work in a luminance-chrominance space (such as $\mathrm{YCbCr}$ ) or a perceptually uniform space (such as CIE Lab), the random decimation prevents us from following this approach. Indeed, the problem is that pixels having missing colors cannot be correctly projected onto a different color space. Therefore, the experiments are conducted in the RGB color space. The dynamics range constraint set $C$ imposes that the pixel values belong to $[0,255]$.

In order to solve Problem (12), we employ the primal-dual $\mathrm{M}+\mathrm{LFBF}$ algorithm recently proposed in [29], which is able to address a wide class of convex optimization problems without requiring any matrix inversion. It offers a good performance and robustness to numerical errors. Its convergence is guaranteed (under weak conditions) and its structure makes it suitable for implementation on highly parallel architectures.

In Fig. 1, we collect the results obtained on the color image airplane $(N=512 \times 512)$ by using the proposed STNLTV (cf. Section 2) and ST-TV [22]. The latter method can be viewed as a particular case of the former one. CCTV and CC-NLTV refer to a channel separable constraint on $\sum_{i=1}^{R} \sum_{\ell=1}^{N}\left\|X_{i}^{(\ell)}\right\|_{p}$, where $X_{i}^{(\ell)}$ denotes the $i$-th column vector of $X^{(\ell)}$ and $\|\cdot\|_{p}$ is the $\ell_{p}$ vector norm. In this example, the bound $\eta$ for each constraint was tuned in order to achieve the best SNR value.

The results demonstrate the interest of considering nonlocal structure tensor measures. Figs. $1 \mathrm{~b}$ and $1 \mathrm{c}$ show the results obtained with CC-TV- $\ell_{2}$ and CC-NLTV- $\ell_{2}$. Although 


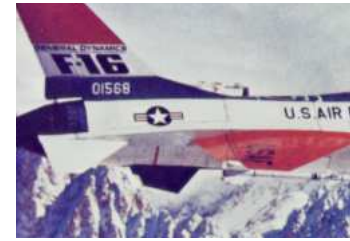

(a) Airplane (zoom)

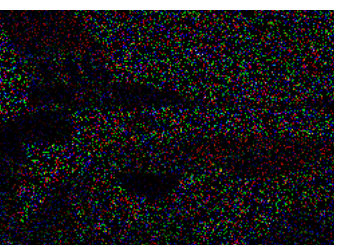

(f) Noisy (zoom)

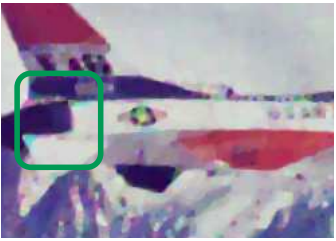

(b) CC-TV- $\ell_{2}: 22.33-0.812$

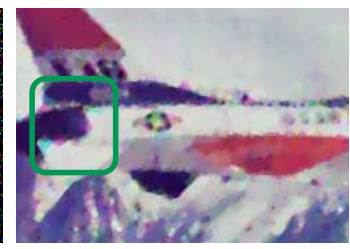

(g) CC-TV- $\boldsymbol{\ell}_{\infty}: 22.00-0.803$

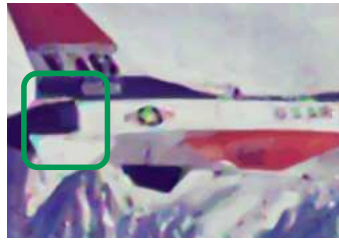

(c) CC-NLTV- $\ell_{2}: 23.20-0.829$

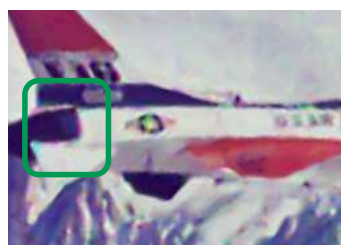

(h) CC-NLTV- $\ell_{\infty}: 23.28-0.827$

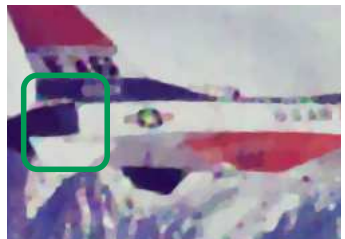

(d) ST-TV- $\ell_{2}: 23.10-0.823$

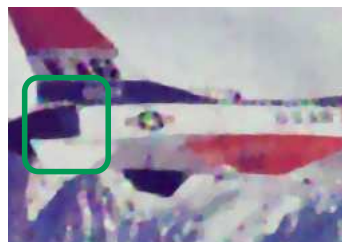

(i) ST-TV- $\ell_{\infty}: 22.68-0.817$

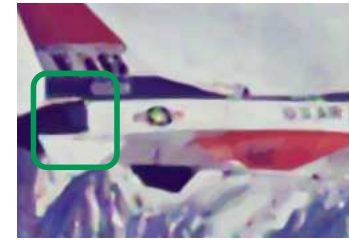

(e) ST-NLTV- $\ell_{2}: \mathbf{2 3 . 6 9}-\mathbf{0 . 8 3 6}$

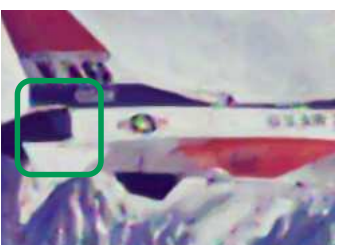

(j) ST-NLTV- $\ell_{\infty}: 23.03-0.823$

Fig. 1. Restored images, along with the corresponding $\mathrm{SNR}_{d B}$ and SSIM values, obtained with different regularizations. The degradation consists of a $3 \times 3$ uniform blur, $90 \%$ of decimation and AWG noise with $\alpha=10$.

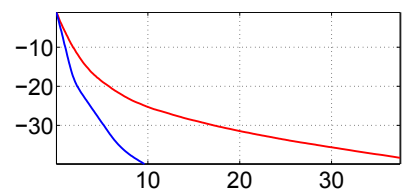

(a) CC-TV- $\ell_{2}$.

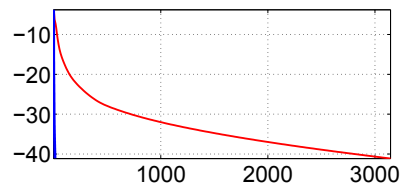

(e) CC-TV- $\ell_{\infty}$.

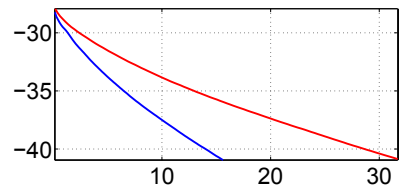

(b) CC-NLTV- $\ell_{2}$.

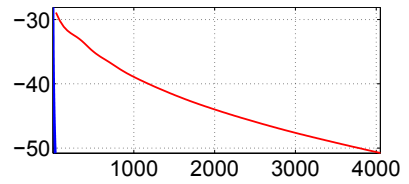

(f) CC-NLTV- $\ell_{\infty}$

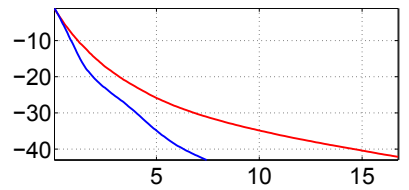

(c) ST-TV- $\ell_{2}$.

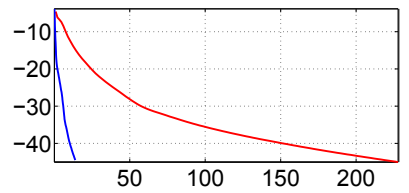

(g) ST-TV- $\ell_{\infty}$.

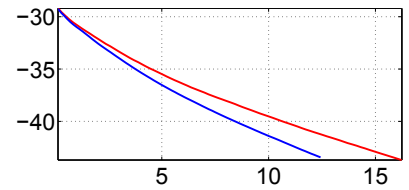

(d) ST-NLTV- $\ell_{2}$.

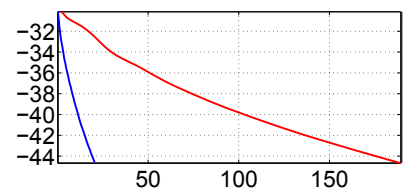

(h) ST-NLTV- $\ell_{\infty}$

Fig. 2. Relative error $\left\|x^{[n]}-x^{[\infty]}\right\| /\left\|x^{[\infty]}\right\|$ vs computational time (in seconds), where $x^{[\infty]}$ denotes the solution computed after a large number of iterations (5000 iterations). Red line: direct projection. Blue line: epigraphical projection.

NLTV better preserves edges and fine details, one can observe a color smearing in the reconstructed images. Figs. 1d and $1 \mathrm{e}$ illustrate the results obtained with ST-TV- $\ell_{2}$ and ST-NLTV- $\ell_{2}$. A significant reduction of color smearing can be noticed. In particular, Fig. 1e shows that combining the structure tensor with NLTV brings the advantages of both methods: preserved details and reduced color smearing. Furthermore, the comparison between the images displayed in the first and second rows indicates that the $\ell_{2}$-norm performs better than the $\ell_{\infty}$-norm. Note that, according to our tests, the nuclear norm leads to poorer results than the $\boldsymbol{\ell}_{\infty}$-norm.

Fig. 2 shows that the epigraphical approach (blue line) leads to a faster convergence than a direct projection method (red line). In particular, for TV- $\ell_{\infty}$ and NLTV- $\ell_{\infty}$, the convergence is about 10 to 40 times faster. The results refer to the airplane image cropped at $256 \times 256$ (a similar behaviour was observed for other images of different sizes). The stopping criterion is set to $\left\|x^{[n+1]}-x^{[n]}\right\| \leq 10^{-4}\left\|x^{[n]}\right\|$ where $\left(x^{[n]}\right)_{n \in \mathbb{N}}$ denotes the sequence generated by M+LFBF. For the $\ell_{1, p}$-ball projectors needed by the direct method, we used the software available on-line [23, 24]. Our codes were developed in Matlab R2011b (the operators $F$ and $F^{\top}$ being implemented in $\mathrm{C}$ using mex files) and all the programs executed on an Intel Xeon CPU at $2.80 \mathrm{GHz}$ with $8 \mathrm{~GB}$ of RAM.

\section{CONCLUSION}

We have proposed a new epigraphical technique for solving constrained convex optimization problems arising in multicomponent image restoration. The obtained results demonstrate the advantages of using the structure tensor and non-local gradients in this context. We have also shown that an $\ell_{1, p}$-norm constitutes a good choice for defining smoothness constraints. Furthermore, our experiments indicate that the epigraphical method converges faster than the approach based on the direct computation of the projections via standard iterative solutions. 


\section{REFERENCES}

[1] D. C. Youla and H. Webb, "Image restoration by the method of convex projections. Part I - Theory," IEEE Trans. Med. Imag., vol. 1, no. 2, pp. 81-94, Oct. 1982.

[2] H. J. Trussell and M. R. Civanlar, "The feasible solution in signal restoration," IEEE Trans. Acous. Speech Signal Process., vol. 32, no. 2, pp. 201-212, Apr. 1984.

[3] P. L. Combettes, "Inconsistent signal feasibility problems: leastsquares solutions in a product space," IEEE Trans. Signal Process., vol. 42, no. 11, pp. 2955-2966, Nov. 1994.

[4] K. Kose, V. Cevher, and A. E. Cetin, "Filtered variation method for denoising and sparse signal processing," in Proc. Int. Conf. Acoust., Speech Signal Process., Kyoto, Japan, Mar. 25-30, 2012, pp. 3329-3332.

[5] T. Teuber, G. Steidl, and R. H. Chan, "Minimization and parameter estimation for seminorm regularization models with I-divergence constraints," Tech. Rep., Technische Universität Kaiserslautern, 2012.

[6] L. Rudin, S. Osher, and E. Fatemi, "Nonlinear total variation based noise removal algorithms," Physica D, vol. 60, no. 1-4, pp. 259-268, Nov. 1992.

[7] G. Gilboa and S. Osher, "Nonlocal Operators with Applications to Image Processing," Multiscale Model. and Simul., vol. 7, no. 3, pp. 1005, 2009.

[8] S. Mallat, A wavelet tour of signal processing, Academic Press, San Diego, USA, 1997.

[9] J.-F. Cai, B. Dong, S. Osher, and Z. Shen, "Image restoration: Total variation, wavelet frames and beyond," J. Amer. Math. Soc., vol. 25, no. 4, pp. 1033-1089, 2012.

[10] G. Peyré, S. Bougleux, and L. D. Cohen, "Non-local regularization of inverse problems," Inverse Problems and Imaging, vol. 5, no. 2, pp. 511-530, 2011.

[11] G. Peyré, "A review of adaptive image representations," IEEE Journal of Selected Topics in Signal Processing, vol. 5, no. 5, pp. 896-911, Sep. 2011.

[12] P. Blomgren and T.F. Chan, "Color TV: total variation methods for restoration of vector-valued images," IEEE Trans. Image Process., vol. 7, no. 3, pp. 304-309, Mar. 1998.

[13] H. Attouch, G. Buttazzo, and G. Michaille, Variational Analysis in Sobolev and BV Spaces: Applications to PDEs and Optimization, MPS-SIAM Series on Optimization, 2006.

[14] C. Zach, T. Pock, and H. Bischof, "A duality based approach for realtime TV-L1 optical flow," in Proceedings of DAGM Symposium on Pattern Recognition, Berlin, Heidelberg, Sep. 12-14, 2007, pp. 214-223, Springer-Verlag.

[15] X. Bresson and T. F. Chan, "Fast dual minimization of the vectorial total variation norm and applications to color image processing," Inverse Prob. Imaging, vol. 2, no. 4, pp. 455-484, Nov. 2008.

[16] V. Duval, J.-F. Aujol, and L. Vese, "Projected gradient based color image decomposition," in Scale Space and Variational Methods in Computer Vision, vol. 5567 of Lecture Notes in Computer Science, pp. 295-306. Springer Berlin / Heidelberg, 2009.
[17] S. Di Zenzo, "A note on the gradient of a multi-image," Computer Vision, Graphics and Image Processing, vol. 33, no. 1, pp. 116-125, Jan. 1986.

[18] G. Sapiro and D. L. Ringach, "Anisotropic diffusion of multivalued images with applications to color filtering," IEEE Trans. Image Process., vol. 5, no. 11, pp. 1582-1586, Nov. 1996.

[19] N. Sochen, R. Kimmel, and R. Malladi, "A general framework for low level vision," IEEE Trans. Image Process., vol. 7, no. 3, pp. 310-318, Mar. 1998.

[20] J. Weickert, "Coherence-enhancing diffusion of colour images," Image and Vision Computing, vol. 17, no. 3-4, pp. 201 - 212, Mar. 1999.

[21] D. Tschumperlé and R. Deriche, "Constrained and unconstrained PDE's for vector image restoration," in Scandinavian Conference on Image Analysis, Bergen, Norway, Jun. 11-14, 2001.

[22] B. Goldluecke, E. Strekalovskiy, and D. Cremers, "The natural vectorial total variation which arises from geometric measure theory," SIAM J. Imaging Sci., vol. 5, no. 2, pp. 537-563, 2012.

[23] E. Van Den Berg and M. P. Friedlander, "Probing the Pareto frontier for basis pursuit solutions," SIAM J. Sci. Comput., vol. 31, no. 2, pp. 890-912, Nov. 2008.

[24] A. Quattoni, X. Carreras, M. Collins, and T. Darrell, "An efficient projection for $\ell_{1, \infty}$ regularization," in International Conference on Machine Learning, Montreal, Quebec, Jun. 1418, 2009, pp. 857-864.

[25] A. Buades, B. Coll, and J. Morel, "A review of image denoising algorithms, with a new one," Multiscale Model. and Simul., vol. 4, no. 2, pp. 490-530, 2005.

[26] G. Gilboa and S. Osher, "Nonlocal linear image regularization and supervised segmentation," Multiscale Model. and Simul., vol. 6, no. 2, pp. 595-630, 2007.

[27] A. Foi and G. Boracchi, "Foveated self-similarity in nonlocal image filtering," in Proc. SPIE, Human Vision and Electronic Imaging XVII, Burlingame, CA, USA, Jan. 22, 2012, vol. 8291, pp. $\mathrm{X}+12$.

[28] G. Chierchia, N. Pustelnik, J.-C. Pesquet, and B. PesquetPopescu, "Epigraphical projection and proximal tools for solving constrained convex optimization problems: Part I," pp. $x+24$, 2012, Submitted. Preprint: http://arxiv.org/pdf/1210.5844.

[29] P. L. Combettes and J.-C. Pesquet, "Primal-dual splitting algorithm for solving inclusions with mixtures of composite, Lipschitzian, and parallel-sum type monotone operators," Set-Valued Var. Anal., vol. 20, no. 2, pp. 307-330, Jun. 2012. 